Supplement of

Quantifying iceberg calving fluxes with underwater noise

O. Glowacki and G. B. Deane

Correspondence to: Oskar Glowacki (oglowacki@ucsd.edu) 
1. Calculating the exposed area at the glacier terminus in pixels squared $\left(A_{\mathrm{img}}\right)$.

The area of the glacier terminus exposed by a selected calving event is determined using the software package ImageJ available free of charge at [https://imagej.nih.gov/ij/download.html]. The analysis proceeds as follows. Firstly, two RGB time-lapse images are identified in the camera data: one taken before the event $\left(i m g_{\mathrm{A}}\right)$, and one taken a time delay $\Delta t=15 \mathrm{~min}$ later $\left(i m g_{\mathrm{B}}\right)$. A difference image is formed by subtracting $i m g_{\mathrm{B}}$ from $i m g_{\mathrm{A}}$ and averaging the color channels leading to a greyscale image $\left(i m g_{C}\right)$ given by:

$$
i m g_{\mathrm{C}}(x, y)=\frac{1}{3} \sum_{i=1}^{3}\left[i m g_{\mathrm{A}, i}(x, y)-i m g_{\mathrm{B}, i}(x, y)\right]
$$

where $(x, y)$ are pixel coordinates and $i$ defines the image color channel (1: red, 2: blue, 3: green). Finally, $A_{\text {img }}$ is found by drawing a polygon around manually identified contrasting space on the glacier terminus in $i m g_{\mathrm{C}}(x, y)$ and then calculating its area in pixels squared. The workflow of the image analysis is illustrated in Figure S1 with a subaerial calving event observed on Aug. 4, 2016.
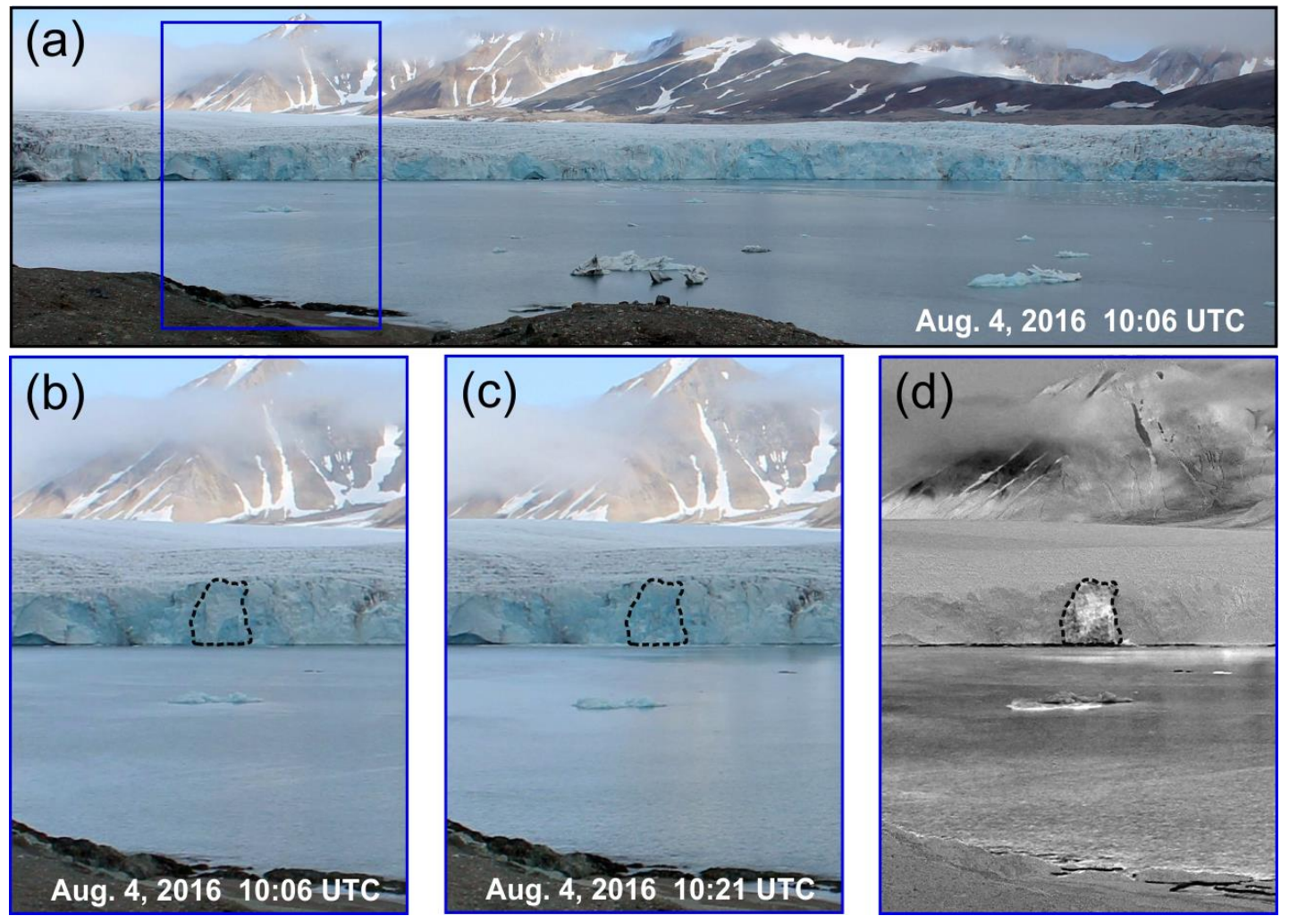

Figure S1: An example of the image processing workflow. Panels (a-c) shows parts of the image of Hans Glacier terminus taken by camera 1 on Aug. 4, 2016 before (a-b, $\left.\boldsymbol{i} \boldsymbol{m} \boldsymbol{g}_{\mathrm{A}}\right)$ and after $\left(\mathbf{c}, \boldsymbol{i} \boldsymbol{m} \boldsymbol{g}_{\mathrm{B}}\right)$ the calving event. The greyscale image resulting from subtraction of $\boldsymbol{i m} \boldsymbol{g}_{\mathrm{B}}$ from $\boldsymbol{i} \boldsymbol{m} \boldsymbol{g}_{\mathrm{A}}\left(\boldsymbol{i} \boldsymbol{m g} \boldsymbol{g}_{\mathrm{C}}\right)$ is shown in panel (d). The area at the glacier terminus exposed by a calving event and manually identified is marked with a black, dashed line. 


\section{Selection of $t_{\text {start }}$ and $t_{\text {end }}$ for the calculation of impact noise energy.}

Calculation of the impact noise energy requires the selection of signal start and stop times, respectively $t_{\text {start }}$ and $t_{\text {end }}$ (see Eq. (4) in the main manuscript). These times were determined as follows. Firstly, we listened to unaltered noise recording and manually noted the start and stop time of the calving signal, which was always clearly distinguishable from the background noise. The times were then checked with a manual review of a low pass filtered version of the recording $\left(f_{\text {cutoff }}=200 \mathrm{~Hz}\right.$ ), which provided an even more unambiguous differentiation between calving and background noise. Both estimates were in good agreement for all analyzed calving events. The filter cutoff frequency was based on a previously observed high correlation between the kinetic energy of the falling ice block and impact noise at frequencies below $200 \mathrm{~Hz}$ (Glowacki et al., 2015). A final check on the time estimates was made by integrating the low pass filtered calving noise over increasing durations, beyond the start and end times, to ensure that the signal was not truncated. Extending the integration time before and after the manually identified event times by 1 or $2 \mathrm{~s}$ produced almost identical results as the original $t_{\text {start }}$ and $t_{\text {end }}$ (differences were typically less than $1 \%$ ). Figure S2 shows an example of the acoustic pressure time series observed during a typical calving event, the median noise level and the selected start and stop times.

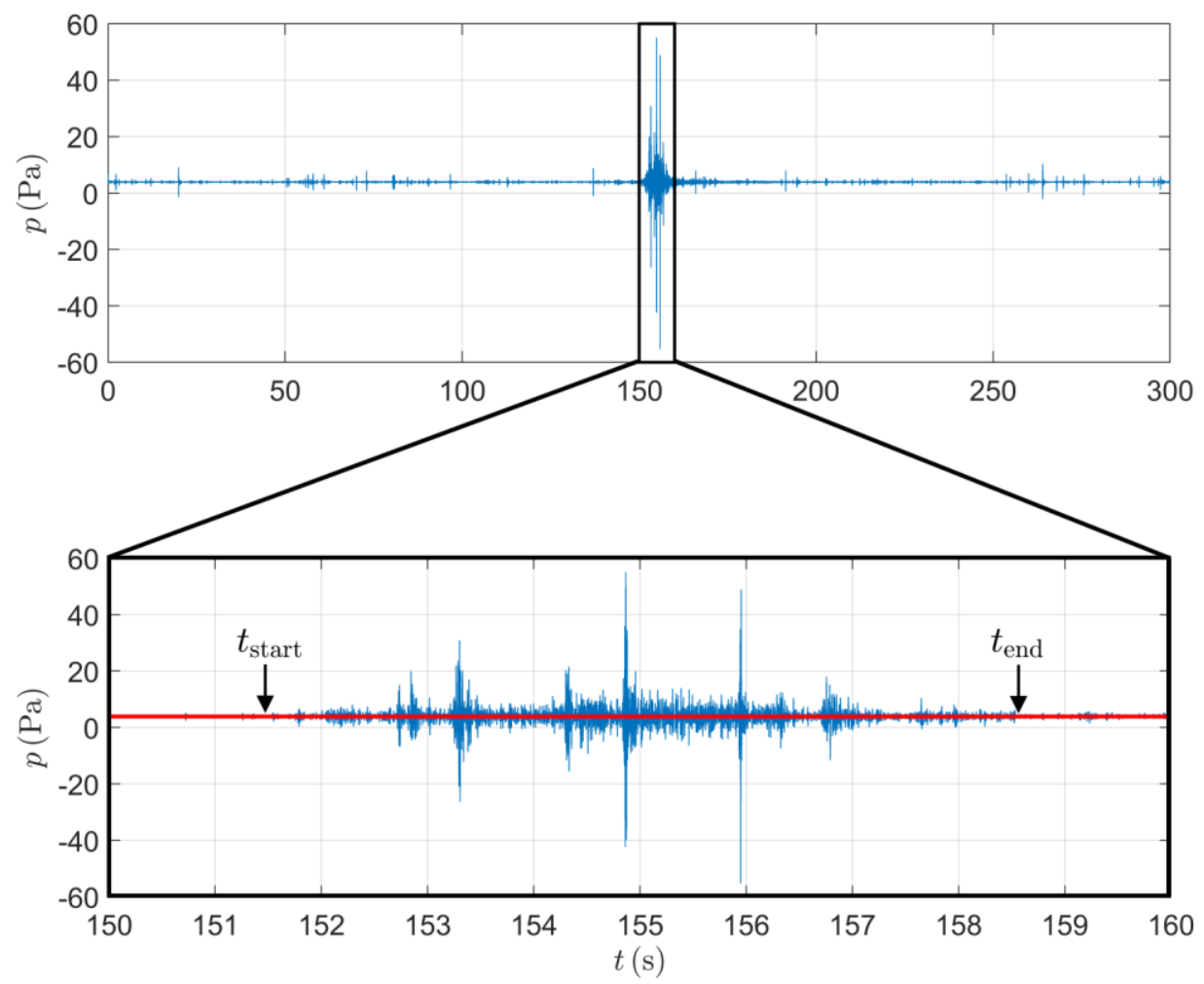

Figure S2: An example of the acoustic pressure recorded at buoy A1 during a calving event. The red line shows median pressure level calculated over a 5 min time period centered on the calving signal. Black arrows point to the locations of $t_{\text {start }}$ and $t_{\text {end }}$, which were used to calculate the impact noise energy. 
3. The loss of acoustic energy generated by iceberg-water impact.

(a) Waveguide propagation of the calving noise from the point of impact to the receiver described using sequence trees of reflections.

Figure S3 shows examples of propagation paths between the iceberg-water impact source and hydrophone receiver. Three boundaries create an acoustic waveguide through which the generated sound travels. There are the ice terminus (' $t$ '), the water surface ('s') and seafloor ('b'). Each of the three boundaries generates a source image (see colored dots in Fig. S3). Isovelocity conditions are assumed to hold so each source image connects with the receiver in a straight line through the point of reflection. The energy contributed by reflected paths depends on the acoustical properties of the medium forming the boundary - its density, sound speed and absorption - and the angle of incidence. These parameters define an angle-dependent reflection coefficient, which can be determined from basic acoustical theory as described below. Acoustic paths with multiple boundary interactions are described using a sequence trees of reflections, including for example surface reflected energy (s), surface-terminus reflected energy (s-t), surface-terminus-bottom reflected energy (s-t-b), etc. (see white box in Fig. S3). These propagation paths could be enumerated and investigated on a path-bypath basis using the method of images to find the total energy loss (see Deane and Buckingham, 1993). However, the analysis here is limited to a comparison between direct-path energy and energy reflected directly from the terminus (see main text).

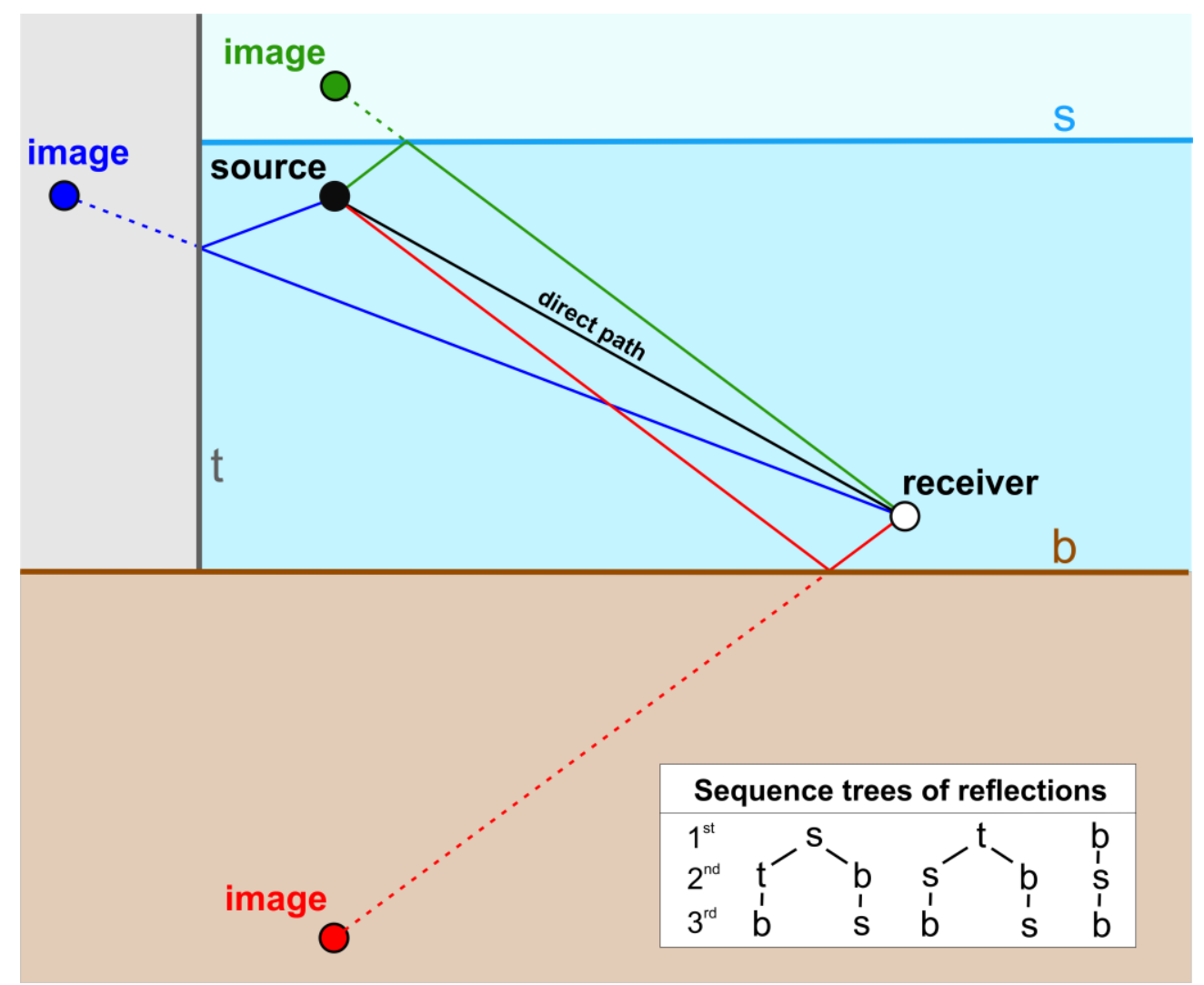

Figure S3: Examples of propagation paths between the sound source (black dot) and receiver (white dot): direct, surface-reflected, icereflected and bottom-reflected. Colored dots show positions of source images in 3 different media creating boundaries for the impact noise: air (green), ice (blue) and seabed (red). There are more possible propagation paths, but all of them include at least 2 or 3 reflections (see scheme on the bottom right), which result in a significantly higher energy loss. 


\section{(b) The influence of terminus-reflected acoustic energy on the received impact noise}

The analysis of the contribution from ice-reflected impact noise to the received acoustic energy proceeds using a wavenumber integration technique. Standard formulas applicable to a fluid-solid interface were used to calculate the angle-dependent Rayleigh ice reflection coefficient $\mathcal{R}$ (see Eqs. 1.61-1.63 in Jensen et al., 2010). The acoustic energy lost through the reflection of the calving noise from the glacier terminus, $R L$, in $\mathrm{dB}$ units is then given by:

$$
R L=-10 \log |\mathcal{R}|
$$

Figure S4a shows the angular dependence of the ice reflection loss for selected compressional $\left(a_{L}\right)$ and shear $\left(a_{L}\right)$ wave attenuation coefficients. The contribution of directly-received and terminus-reflected impact noise was calculated using a wavenumber integration technique (see Eq. 4.3.2 in Brekhovskikh and Lysanov, 1982). Results of this analysis are shown in Figure S4c and discussed in detail in the main manuscript (see subsection 3.4.2).
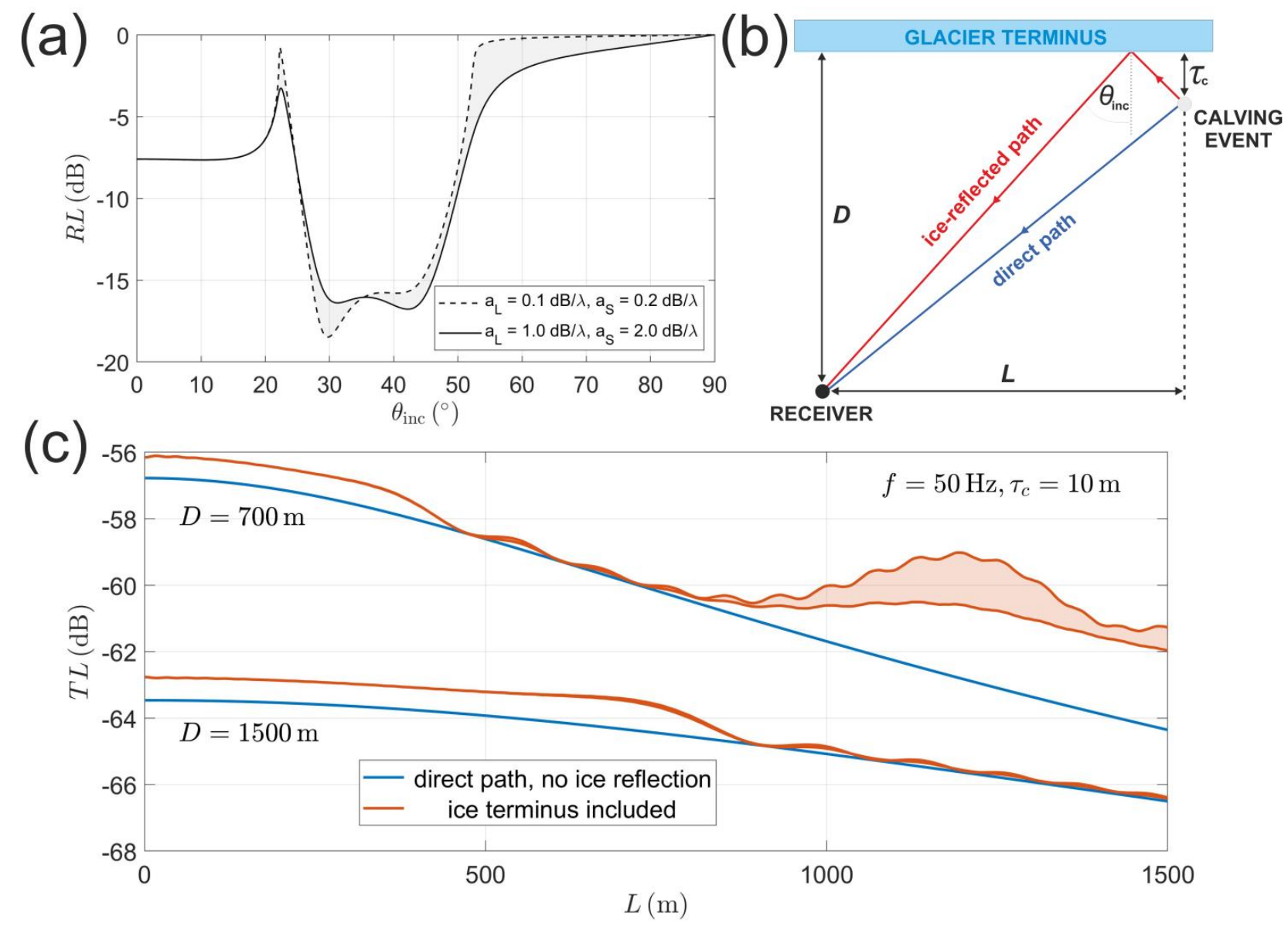

Figure S4: (a) Angle-dependence of ice reflection loss in dB units, (b) a scheme of the noise transmission for direct path and ice-reflected path, and (c) transmission loss in $\mathrm{dB}$ with (red) and without (blue) terminus reflection for changing location of the calving event and distance to the glacier. Shaded areas indicate different $\boldsymbol{T} \boldsymbol{L}$ values for different absorption coefficients. Reflections from the ocean bottom and sea surface are not included in this analysis. A reflection loss of $\mathbf{- 5 , - 1 0}$ and $\mathbf{- 1 5} \mathrm{dB}$ corresponds, respectively, to a factor of 3,10 and 32 in energy loss. 


\section{(c) Total energy loss of the impact noise}

The total energy loss estimated using Bellhop for each calving event for the entire inventory of 169 events ranges from -57 to $-47 \mathrm{~dB}$ (Figure S5). Three major factors affecting the transmission loss are included in this analysis: fluctuations in thermohaline structure, variability of the ocean depth along the waveguide and reflection of the calving noise energy from the glacier terminus. The contribution of the latter was estimated to be between 0 and 2 $\mathrm{dB}$ for each event (see section 3.4.2 in the manuscript and Fig. S4). A maximum absolute difference of around 5 $\mathrm{dB}$ between the median energy loss calculated for different calving events results mainly from the large variability in bathymetry profiles along the propagation paths (see Fig. 3 in the manuscript).

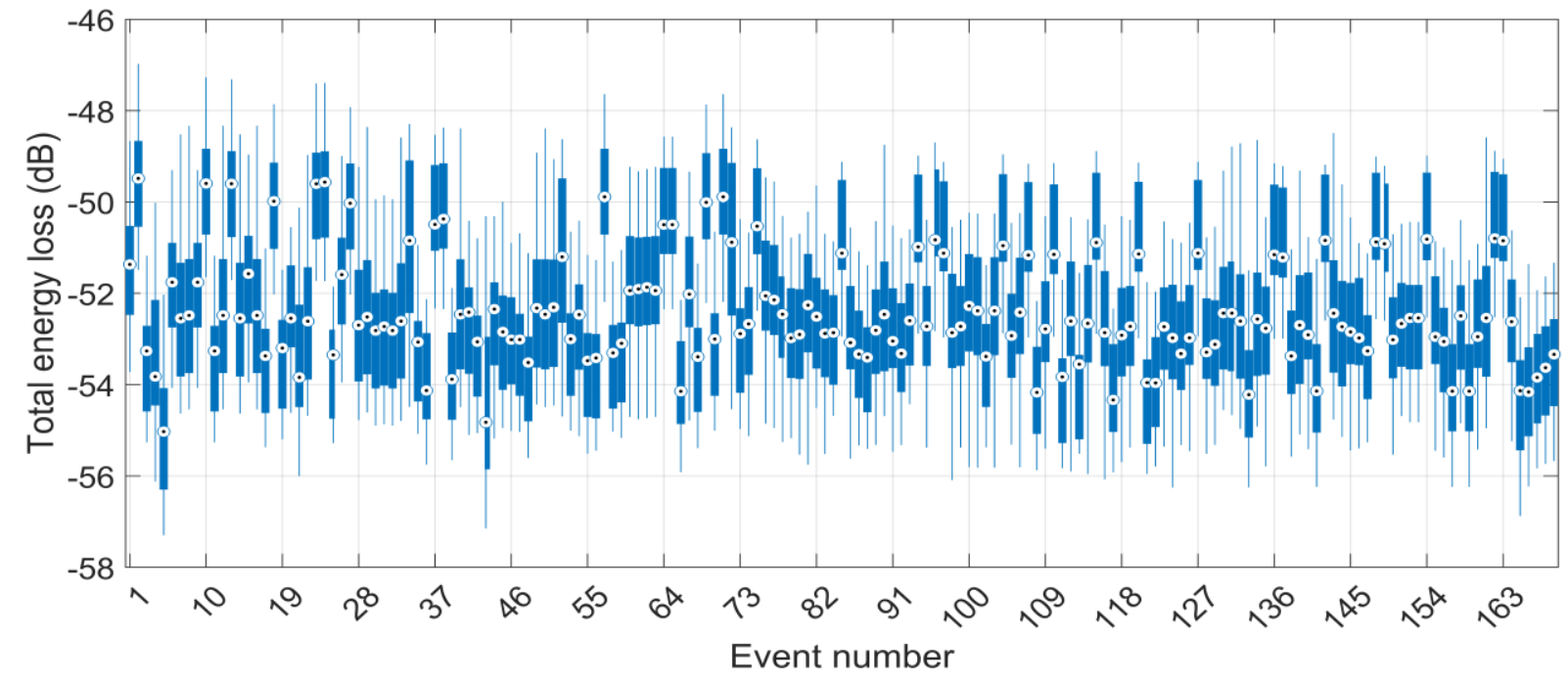

Figure S5: Total loss of energy in $\mathrm{dB}$ units computed for all 169 calving events, including both transmission loss in a waveguide and reflections from the glacier terminus. A loss of $-52 \mathrm{~dB}$ corresponds to a factor of $6.3 \times 10^{-6}$ in the energy at source. White points with small dots indicate median values. 
4. Relationship between the impact energy and acoustic emission

(a) Inclusion of outliers

Figure S6 shows relationship between the block-water impact energy and resulting acoustic emission below $100 \mathrm{~Hz}$ with the inclusion of two outliers. The energy conversion efficiency, $\eta$, and exponent of the power law, $b$, estimated for all 169 calving events equals $5.66 \times 10^{-7}$ and 0.94 , respectively.

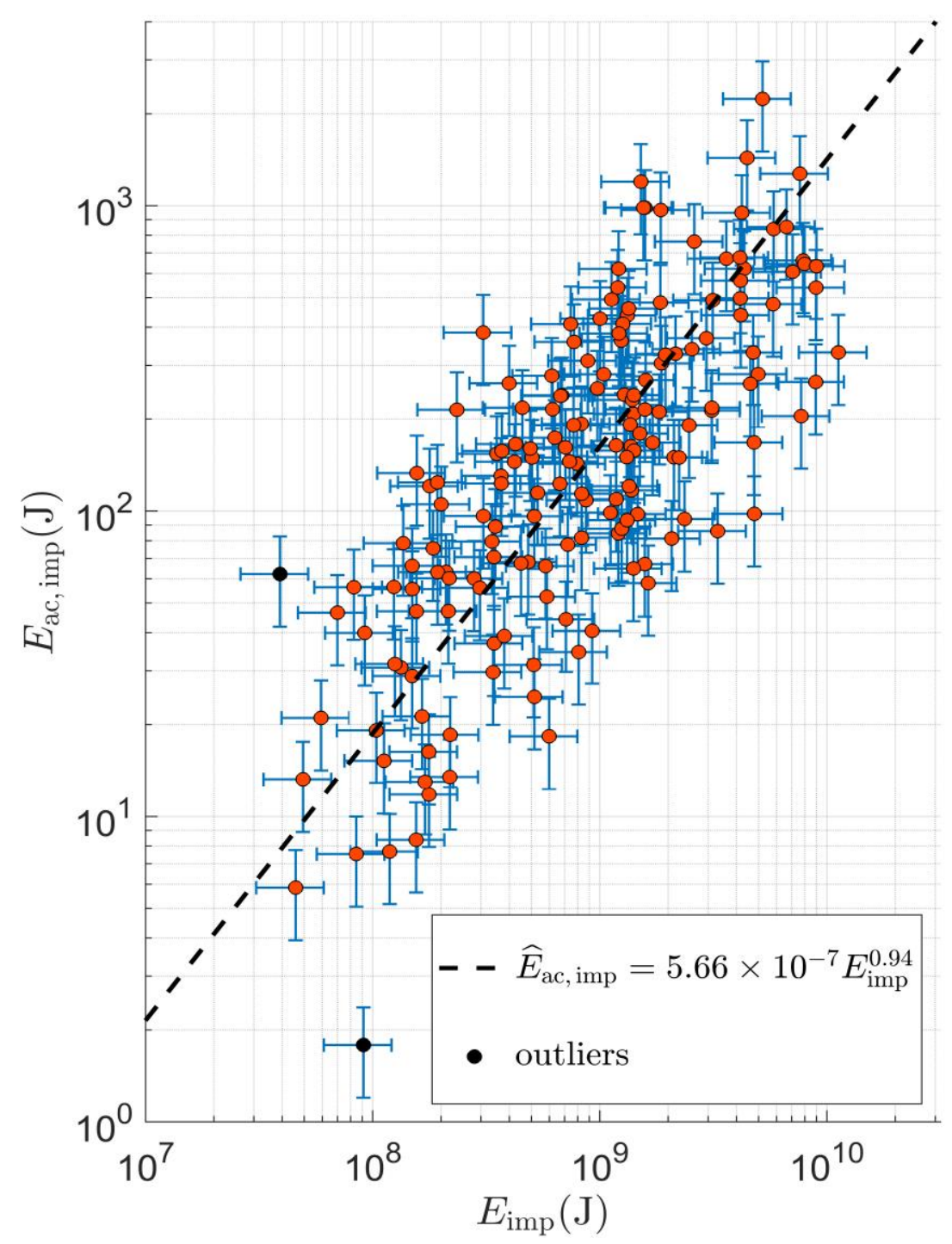

Figure S6: Relationship between the block-water impact energy and underwater acoustic emission below $100 \mathrm{~Hz}$. Uncertainties are marked with blue whiskers and were estimated to be $33.0 \%$ for both variables. The remaining scatter in impact energy is most likely caused by different calving styles and an associated variability in source mechanisms. Two outliers are included in this analysis and marked with black dots. 
(b) Comparison with Glowacki et al. (2015)

Figure S7 shows the relationship between impact noise energy measured from the images, $E_{\text {imp }}$, and calculated from the noise energy, $\hat{E}_{\mathrm{imp}}$, using the energy conversion coefficients derived in this study and those presented by Glowacki et al. (2015). Glowacki et al. (2015) assumed simple cylindrical spreading loss, which underestimated the impact noise energy and resulted in a lower energy conversion efficiency $\eta$ than the value presented here.

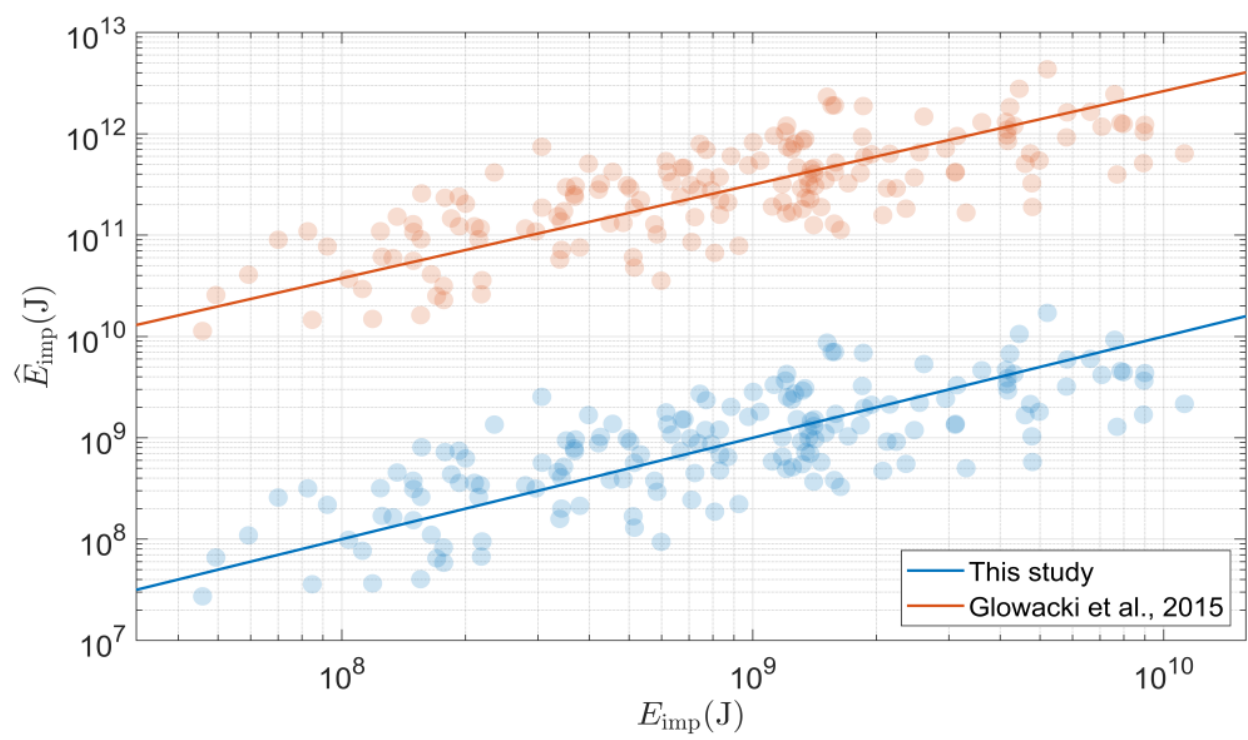

Figure S7: Comparison between measured and modeled impact energy computed based on (blue) this study and (red) using the energy conversion efficiency reported in Glowacki et al. (2015). The ratio of 300:1 in acoustic energy corresponds to the increase of noise power by almost $25 \mathrm{~dB}$.

\section{References}

Brekhovskikh, L., and Lysanov, Y.: Fundamentals of Ocean Acoustics, Springer-Verlag, New York, https://doi.org/10.1007/978-3-662-02342-6, 1982.

Deane, G. B., and Buckingham, M.: An analysis of the three-dimensional sound field in a penetrable wedge with a stratified fluid or elastic basement, J. Acoust. Soc. Am., 93(3), 1319-1328, https://doi.org/10.1121/1.405417, 1993.

Glowacki, O., Deane, G. B., Moskalik, M., Blondel, Ph., Tegowski, J., and Blaszczyk, M. (2015), Underwater acoustic signatures of glacier calving, Geophys. Res. Lett., 42, 804-812, https://doi.org/10.1002/2014GL062859, 2015 .

Jensen, F. B., Kuperman, W. A., Porter, M. B., Schmidt, H.: Computational Ocean Acoustics, Springer Science+Business Media, LLC, New York, https://doi.org/10.1007/978-1-4419-8678-8, 2011. 